\title{
Application of Full Digital Simulation Test Environment in Aerospace Software Testing
}

\author{
Xue Shuai, Zhao Long, Hu Xiaoxi \\ Beijing Institute of Aerospace Control Devices, Beijing, China
}

Email address:

chenpeng_top@163.com (Chen Peng),Pengwan1124@163.com (Wan Peng),jpxu@seu.edu.cn (Xu Jinping)

\section{To cite this article:}

Xue Shuai, Zhao Long, Hu Xiaoxi. Application of Full Digital Simulation Test Environment in Aerospace Software Testing. Science Discovery. Vol. 7, No. 1, 2019, pp. 56-60. doi: 10.11648/j.sd.20190701.22

Received: April 20,2019; Accepted: May 20, 2019; Published: May 23, 2019

\begin{abstract}
With the significant increase in the degree of informationization of spacecraft, higher requirements are placed on the reliability of aerospace software. Therefore, the position of software testing in the software development cycle is particularly important. The current software testing environment has significant limitations for achieving high reliability and high quality requirements for aerospace software. In order to solve this problem and further improve the quality of software testing, this paper uses the full digital simulation test environment to test the aerospace software. This paper first analyzes the current test conditions and shortcomings of the aerospace software, then introduces the basic concept of the full digital simulation test environment, and finally applies the full digital simulation test environment to the aerospace model software test, improving the quality and efficiency of software testing. The application results show that the full digital simulation test environment has stronger versatility and flexibility than other software test environments, and is more suitable for current aerospace software testing.
\end{abstract}

Keywords: Aerospace Software, Full Digital Simulation Test Environment, Software Testing

\section{全数字仿真测试环境在航天型号软件测试中的应用}

薛帅, 赵龙, 胡晓曦

北京航天控制仪器研究所, 北京, 中国

邮箱

chenpeng_top@163.com（陈鹏）, Pengwan1124@163.com（万蓬）, jpxu@seu.edu.cn（徐金平）

\begin{abstract}
摘要: 航天器信息化程度大幅提升, 对航天型号软件的可靠性提出更高要求, 因此软件测试在软件研制周期中的地位 就显得尤为重要。对于实现航天型号软件的高可靠性和高质量要求, 当前软件测试环境还存在很大的局限性。为了解 决该问题, 并进一步提高软件测试质量, 本文采用全数字仿真测试环境对航天型号软件展开测试工作。本文首先分析 了当前航天型号软件现有测试条件及其不足, 然后介绍了全数字仿真测试环境的基本概念, 最后将全数字仿真测试环 境实际应用在航天型号软件测试中, 提高了软件测试的质量和效率。应用结果显示, 全数字仿真测试环境与其他软件 测试环境相比有着较强的通用性及灵活性, 更适用于目前的航天型号软件测试。
\end{abstract}

关键词: 航天型号软件, 全数字仿真测试环境, 软件测试 


\section{1. 引言}

随着我国航天事业的发展, 航天器的结构和功能日益 复杂, 信息化程度也越来越高, 很多重要和复杂的功能都 需要通过软件来实现, 因此软件在航天任务中起到了举足 轻重的作用。航天型号软件能否安全可靠地运行, 直接影 响整个航天任务能否顺利完成以及参与人员的生命安全。 近些年来, 国内外出现越来越多因为软件出现故障而失败 的航天任务。如何保证航天型号软件的安全性和可靠性, 进而保证航天任务的圆满成功, 成为了航天工程师急需解 答的课题。

通过分析航天型号软件失效的案例, 可以发现软件发 生故障的一个最主要的原因就是测试不够充分, 不能够发 现一些隐藏很深的关键性问题[1]。航天型号软件具有嵌入 式、实时性高、依赖硬件强等特点, 所以在进行航天型号 软件测试时, 往往需要在真实的硬件环境下进行。然而这 些硬件设备通常研制时间非常长、研制的数量又很少，而 价格往往非常昂贵, 导致软件的开发进度常常受制于硬件 的研制, 造成软件开发周期大大延长, 进而也给软件测试 带来了极大的困难[2-4]。

\section{2. 航天型号软件现有测试条件及不足}

目前航天型号的研发流程, 基本上都是先进行总体的 系统设计, 再进行硬件的设计工作以及硬件样机的生产, 然后才是软件的设计、开发和测试工作，以及最后的软硬 件的系统联试和验证, 如图1所示。

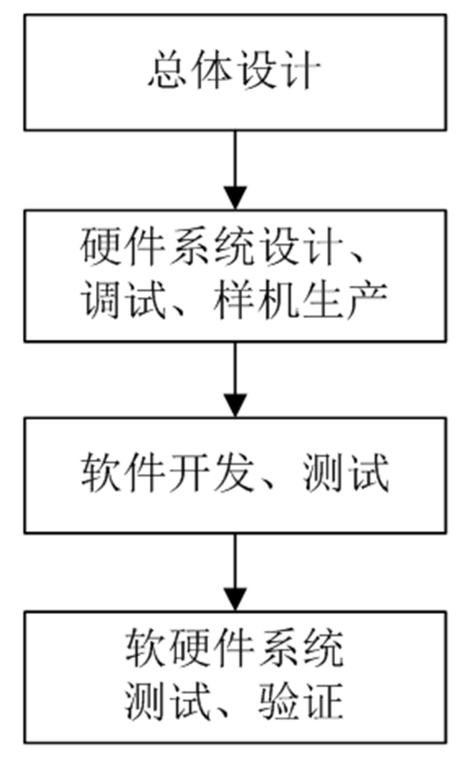

图1 当前航天型号研发流程。

正因为这样的研发流程, 导致航天型号软件与物理硬 件有着极大的关联性, 软件的验证与测试工作很大程度上 依赖于通过接口连接的硬件设备, 因此在对航天型号软件 进行测试时, 使用的测试环境通常为全物理测试环境或半 物理仿真测试环境 $[5,6]$ 。

全物理测试环境, 顾名思义就是将被测软件运行在真 实的目标环境中, 通过接口与软件相连接的也均为真实的
硬件设备。常用的测试工具有总线分析器、逻辑分析仪等。 全物理测试环境的主要优势是被测软件具有真实的运行 环境, 测试结果可信度高。

半物理仿真测试环境是将被测软件运行在真实的目 标硬件上, 其余的外部环境条件, 如通过接口传入的数据 等, 都是通过软件仿真实现的。这样的测试环境通常由目 标硬件和测试计算机组成。目标硬件是用于运行被测软件, 同时还能够进行断点调试, 并给出软件运行情况等功能。 测试计算机则是用来进行仿真外部环境条件, 提供外部数 据输入和激励, 同时完成测试管理及测试结果的记录与分 析。半物理仿真测试环境是当前航天型号软件最为常用的 测试环境[7]。

以上的两种软件测试环境都是基于真实目标硬件的 测试环境，但是在使用中存在以下问题:

（1）由于与硬件设备的紧密性，航天型号软件的设 计与开发通常需要等待硬件设备的研制完成, 并且软件研 制完成后除了设计师要进行软件调试外, 还需要进行开发 方测试、第三方测试、系统联试等一系列工作，导致软件 研制的过程需要占用硬件的时间多, 使得整个项目时间紧、 任务重，造成整个航天型号研制的周期大大延长;

（2）由于硬件设备的产量很少或是硬件设备价格高 昂, 导致在航天型号软件的调试与测试阶段中, 进行有关 硬件失效的故障注入极为困难, 甚至是不可能的, 这样也 就使软件中的异常处理设计无法得到验证与测试;

(3) 由于被测软件运行在真实的物理环境中, 使得 在航天型号研制的早期阶段, 当出现问题时, 往往无法区 分出到底是因为硬件设备出现的问题还是软件出现的问 题;

（4）由于航天型号软件基本上均为嵌入式软件, 对 嵌入式软件的运行情况进行监控是比较困难的, 给测试结 果的采集与记录工作造成很大的困难, 也导致了问题复现 的难度大大增加，难以对问题进行精确定位;

（5）进行非干预性测试难度大。非干预性测试就是 在进行测试的过程中, 所采取的测试技术和手段不能对被 测软件的执行情况产生任何影响。比如, 在进行时间性能 测试和覆盖率信息统计的时候, 往往要通过代码插桩的手 段来实现, 这样就需要修改被测软件的源代码, 就会导致 代码量膨胀。由于航天型号软件实际使用的代码空间就极 其有限, 这样处理后的代码往往会无法加载, 测试就无法 有效地进行[8]。

\section{3. 全数字仿真测试环境}

全数字仿真测试环境就是通过再现计算机硬件行为 的方式, 对硬件设备的软件模拟, 一般采用传统的计算机 程序设计语言实现。全数字仿真测试环境通过对嵌入式软 件实际运行环境的模拟, 使得嵌入式软件可以毫不修改就 能够运行在使用普通计算机搭建起来的模拟仿真环境中, 并且能够监控嵌入式软件运行的全过程, 执行已经设计好 的测试用例, 实现测试嵌入式软件的目的 [9]。

全数字仿真测试环境对嵌入式软件实际运行环境的 模拟可以主要分成两大部分, 即目标硬件环境的模拟和目 标数据环境的模拟 $[10,11]$ 。目标硬件环境的模拟是指嵌入 
式软件运行所必需的硬件设备, 包括对CPU和内存的模拟, 以及其他串口、总线和芯片的仿真都是目标硬件环境模拟 的主要内容。目标数据环境的模拟就是指对嵌入式软件运 行时所需要的外部数据源的模拟, 这些数据源可能是陀螺 仪、压力传感器等输出的数据, 一般通过接口通信的方式 传输给嵌入式软件进行处理。

一般的全数字仿真测试环境的构成如图2所示, 主要 可以分成以下几个模块: 硬件模型仿真模块 (机械结构仿 真、外部环境仿真等) 、被测软件CPU仿真模块 (CPU内 核仿真、1553B仿真、串口仿真等) 、地面控制系统仿真 模块、与被测软件有关的其他系统仿真 $[12,13]$ 。这些模块 通过通信接口与全数字仿真测试环境运行内核进行关联。 测试人员的操作, 如控制指令、测试用例, 通过人机界面 层以及相连的运行内核传达给各个模块。测试人员通过在 人机界面层的各种操作, 来完成各种测试和调试工作, 例 如测试脚本的运行、源代码的调试、覆盖率信息统计、软 件状态信息的显示、遥测量的显示、遥控数据生成及保存 等等。

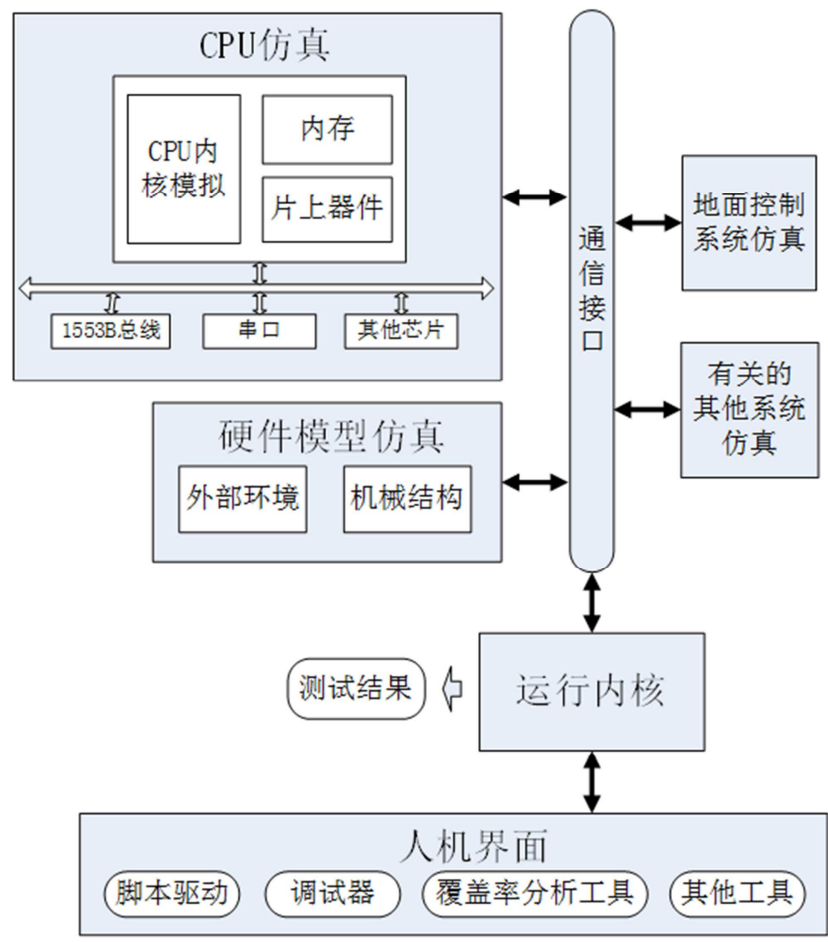

图2 全数字仿真测试环境的构成。

\section{4. 全数字仿真测试环境的应用}

以某航天型号某系统监测软件的测试过程为例对全 数字仿真测试环境的应用进行说明, 使用全数字仿真平台 进行测试主要分为以下四个过程: 被测软件总体架构分析、 外设接口的模拟、测试脚本的编写和运行以及覆盖率数据 统计。下面对主要测试过程进行介绍。

\section{1. 被软件总体架构分析}

对被测软件总体架构的分析是整个测试过程中首要 并且是最关键的步骤, 这直接关系到后续外设接口的实现 以及测试结果的可信性。在这个步骤中主要关注的是被测 软件的CPU、寄存器、中断和内外存储单元等。在被测软 件使用的内核方面, 要确定被测软件使用的CPU的类型以 及主频, 需要关注CPU主频是因为要用来模拟出被测软件 实际的时间性能。其次要分析出被测软件的外设接口的实 现架构, 包括被测软件使用了哪些串口、通信总线, 连接 了哪些外设, 以及中断的实现情况。本例被测软件的总体 架构如图3所示。

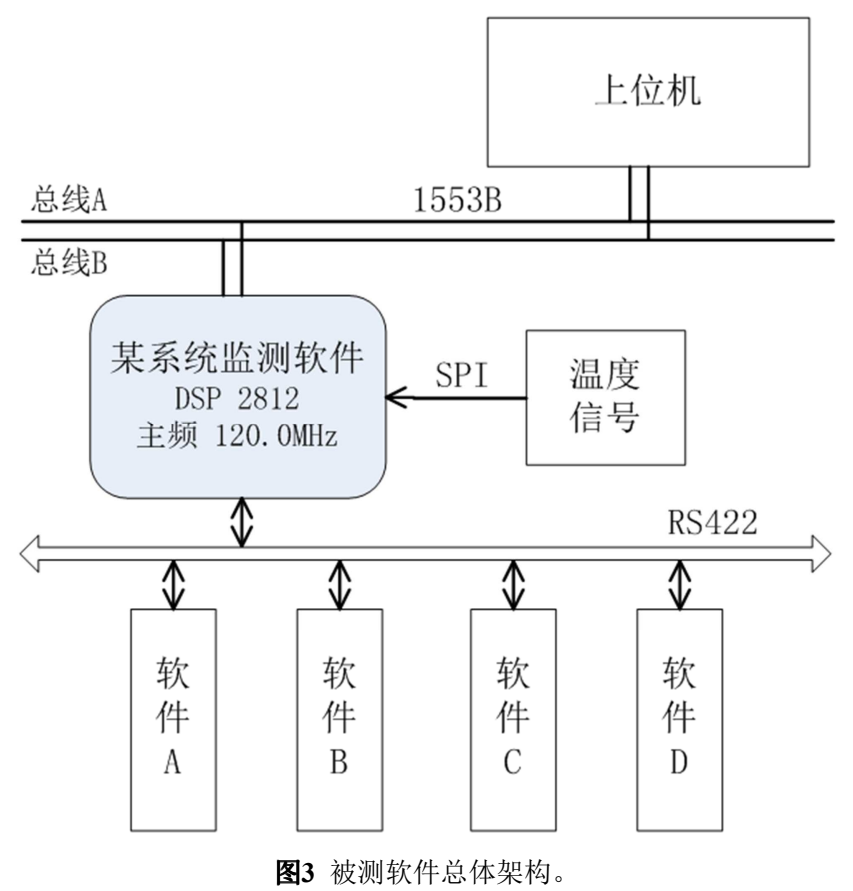

在分析完被测软件的总体架构后, 测试人员通过实现 每个外设模块的模拟, 就可以搭建一整套的仿真测试环境。 将被测软件运行在搭建好的测试环境中, 就能完全模拟出 软件在真实硬件上的执行过程和运行状态, 随后便可在此 基础上进行软件的测试。

\section{2. 外设接口的模拟}

外设接口的模拟, 是保证全数字仿真测试环境准确运 行最重要的一环。这是因为硬件实物测试环境的接口数据 是通过真实的外设传入的, 而全数字仿真测试环境的接口 数据是通过编写的外设接口模块仿真而来, 如果不能够准 确模拟传入数据的数据结构和时间特性, 就会使仿真的结 果偏离实际情况。

在对外设接口进行模拟时, 主要考虑输入|输出口以 及中断的实现方式。在本例中, 被测软件通过RS422串口 与其他 4 个被监测软件进行数据通信, 通过 1553 B总线与上 位机连接用于控制指令的接收, 并使用SPI接口接收温度 信号。在实现外设接口模拟的时候, 不仅要考虑如何监测 软件的数据输出, 同时也要考虑外设通过这些接口对被测 软件进行数据输入时的中断触发方式。 
为提高外设接口模拟的准确性, 可以在长期测试过程 中不断积累经验, 将常用芯片进行封装, 提高外设接口仿 真的准确性, 同时缩短仿真测试环境的搭建周期。

\section{3. 测试脚本的编写和运行}

对软件进行测试, 实际上就是设计测试用例, 并依据 测试用例执行软件的过程。通过软件实际执行结果与预期 情况的对比, 来评估软件实现的功能是否正确。

测试用例在全数字仿真测试环境中的实现是通过执 行测试脚本来完成的。在编写测试脚本时, 要考虑被测软 件实际运行的流程, 比如在每一个时间节点应该有哪些接 口数据的注入, 上位机在哪个时间节点发送控制指令, 应 该监测哪些状态量等等。

\section{4. 覆盖率数据统计}

由于航天型号软件的高可靠性、高安全性的要求, 进 行软件测试覆盖率统计工作就显得十分必要。通过对软件 测试中的代码覆盖率进行统计, 可以分析出被测软件中哪 些代码未被执行, 为补充测试用例、提高测试完整性提供 依据。全数字仿真测试环境中的覆盖率统计工具可以在每 个测试用例执行完成后, 生成一个代码覆盖率文件, 并在 所有测试用例执行结束后, 将所有生成的覆盖率文件进行 叠加, 最后生成总的覆盖率统计结果。通过分析总体覆盖 率统计结果, 找出未被覆盖代码未能执行的原因, 可以看 出到底是测试用例设计缺失还是软件设计问题, 进而发现 软件深层次的问题。

\section{5. 应用效果分析}

通过全数字仿真测试环境在多个航天型号软件测试 中的应用, 可以总结出其有以下几点优势:

(1) 软件研制过程可脱离硬件实施。全数字仿真测 试环境主要采用软件模拟硬件的方式, 将嵌入式软件和硬 件两者剥离开来, 由全数字仿真测试环境来给嵌入式软件 提供运行环境以及各类接口, 使得软件的调试、开发方测 试、甚至第三方测试等大部分过程（功能测试及验证、接 口测试及验证、边界测试、安全性测试、可靠性测试、逻 辑测试等) 均可脱离硬件开展实施, 可以减少软件研制过 程中对硬件的占用时间 $70 \%$ 以上。

(2) 进一步提高测试覆盖性。在软件调试以及测试 过程中往往很多时候由于硬件的限制导致测试用例无法 完全执行, 例如硬件接口的损坏功能、无法通过硬件达到 的边界值等, 但是通过采用全数字仿真测试环境可以解决 上述问题, 保证软件测试的覆盖性。

(3) 有利于问题的定位与解决。在使用硬件实物测 试环境时, 进行的测试基本上是黑盒测试, 被测软件的运 行状态难以全面捕捉, 当出现问题时, 很难定位与解决。 而使用全数字仿真测试环境进行测试是一种白盒测试, 在 测试的过程中可以进行断点调试, 极大地方便了问题的定 位与解决。

同时, 也可以看出全数字仿真平台的不足：由于不是 基于真实测试环境的测试, 一些如性能测试、余量测试等
无法脱离硬件实施的测试项目, 仍需要在硬件实物测试环 境下进行。

\section{5. 结论}

通过对全数字仿真测试环境在实际应用情况的分析, 可以看出全数字仿真测试环境和全 $\mid$ 半物理测试环境相比 有着较强的通用性、灵活性, 通过脚本中的数据输入, 可 以模拟出各种不同的激励环境, 实现硬件设备不能实现的 故障模式或并发输入, 进一步提高了软件测试的覆盖性。 同时, 全数字仿真测试环境也使得航天型号软件在开发与 测试时能够摆脱对硬件环境的依赖, 能够显著缩短航天型 号的整体研制周期。

总之, 全数字仿真测试环境为航天型号软件测试提供 一个切实可行的技术途径, 为最终实现航天型号工程软、 硬件的并行开发与测试迈出了关键的一步。

\section{参考文献}

[1] 侯成杰. 国外航天软件故障原因分析 [J]. 航天器工 程,2012,21(1):89-96。

[2] Sharp D C, Bell A E, Gold J J, et al. Challenges and solutions for embedded and networked aerospace software systems[J]. Proceedings of the IEEE, 2010, 98(4): 621-634.

[3] 侯聪聪. 嵌入式计算机软件测试关键技术 [J].电子技术与软 件工程,2018(11):214-214。

[4] 姚佳瑜.嵌入式箭载计算机飞行控制软件测试技术研究[D]. 上海师范大学, 2014。

[5] 解志君.嵌入式软件测试技术分析研究[J].自动化与仪器仪 表,2016(12):3-4。

[6] 黎晖,石小华,林柯军,等.工程装备嵌入式软件测试环境平台 技术研究[J].计算机测量与控制,2016(4): 3。

[7] Xu W, Yang W, Li C, et al. Research on Embedded and Monitoring Test Technology of $\mathrm{C} 44$ ISR System[C]/International Conference on Human Centered Computing. Springer, Cham, 2018: 86-92.

[8] 姚佳瑜.嵌入式软件中断系统的测试[J].电子技术与软件工 程,2018(9):40-40。

[9] Gao F, Deng F, Yan Y, et al. Full Digital Simulation Testing of Networked Embedded Software[C]//2018 IEEE International Conference on Software Quality, Reliability and Security Companion (QRS-C). IEEE, 2018: 45-50.

[10] He Y, Zhang F. Research on Embedded Software Testing Technology Based on Full Digital Simulation[J]. Science Mosaic, 2015.

[11] Klepper J, Knapke R, Sirbaugh J, et al. Computational Modeling and Simulation Applications to Support Integrated Test and Evaluation (IT\&E) at AEDC[C]//2018 Aerodynamic Measurement Technology and Ground Testing Conference. 2018: 3873 . 
[12] 陈㻦,罗永红,李春雷.基于QEMU的全数字仿真环境设计 $[\mathrm{J}]$. 环境技术,2016(4):13。
[13] 李昌,邓矢斧,冯雷,等.基于全数字的航空机载软件验证平台 研究 [J].计算机测量与控制,2018(6):34。 